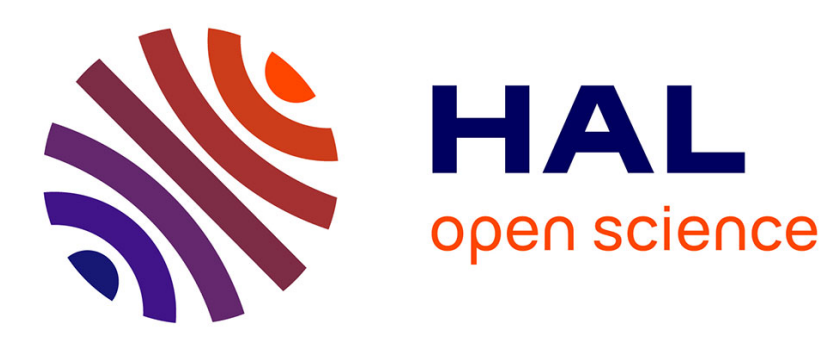

\title{
Designing an Expandable Illuminated Ring to Build an Actuated Ring Chart
}

\author{
Maxime Daniel, Guillaume Rivière, Nadine Couture
}

\section{To cite this version:}

Maxime Daniel, Guillaume Rivière, Nadine Couture. Designing an Expandable Illuminated Ring to Build an Actuated Ring Chart. TEI 2018 Work in Progress, 12th International Conference on Tangible, Embedded and Embodied Interactions, Mar 2018, Stockholm, Sweden. pp.140-147, 10.1145/3173225.3173294 . hal-01668076

\section{HAL Id: hal-01668076 https://hal.science/hal-01668076}

Submitted on 10 Dec 2018

HAL is a multi-disciplinary open access archive for the deposit and dissemination of scientific research documents, whether they are published or not. The documents may come from teaching and research institutions in France or abroad, or from public or private research centers.
L'archive ouverte pluridisciplinaire HAL, est destinée au dépôt et à la diffusion de documents scientifiques de niveau recherche, publiés ou non, émanant des établissements d'enseignement et de recherche français ou étrangers, des laboratoires publics ou privés. 
TEI'2018 (C) ACM, 2018. This is the author's version of the work. It is posted here by permission of ACM for your personal use. Not for redistribution. The definitive Version of Record was published in the Proceeding of the Twelfth International Conference on Tangible, Embedded, and Embodied Interaction, pp. 140-147,

http://dx.doi.org/10.1145/3173225.3173294

\section{Designing an Expandable Illuminated Ring to Build an Actuated Ring Chart}

\author{
Maxime Daniel $^{1,2}$ \\ ${ }^{1}$ ESTIA, Estia-Recherche \\ F-64210 \\ 64210, Bidart, France \\ m.daniel@estia.fr
}

\author{
Guillaume Rivière ${ }^{1,2}$ \\ ${ }^{2}$ Univ. Bordeaux, CNRS, LaBRI, \\ UMR 5800, F-33405 \\ 33405, Talence, France \\ g.riviere@estia.fr
}

\begin{abstract}
Data physicalizations are growing popular in many societal domains which indicates a strong potential for fostering public engagement thanks to public exhibition (e.g. in train stations, in airports, on roundabouts, and in enterprises). We focus on dynamic physical charts for visualizing renewable energy forecasts in public spaces. To get charts readable from any point of view around, we propose a physical ring chart inspired by stone cairns. To build this physical chart, we designed an expandable and stackable illuminated ring. In this paper, we describe the design process and the limitations of the first prototype of such a ring.
\end{abstract}

\section{Author Keywords}

Tangible User Interfaces; Data Physicalization; ShapeChanging Interfaces; Actuation; Dynamic Physical Ring Charts; Renewable Energy Forecast; Shifting Energy Demand; Public spaces.

\section{Introduction}

Tangible User Interfaces "give physical form to digital information, employing physical artifacts both as representations and controls for computational media" [16]. Recently, Data Physicalization has emerged as a new research area at the intersection of Data Visualization and Tangible User Interfaces. In 2015, Jansen et al. [7] defined 


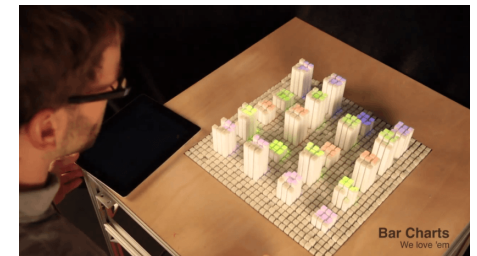

Figure 1: Bar charts displayed with InFORM [3].

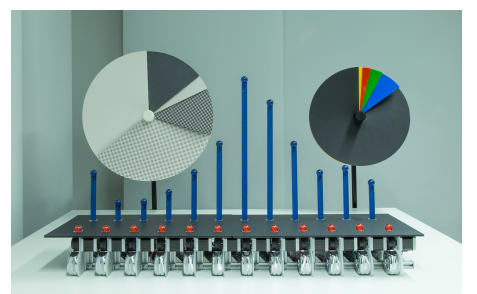

Figure 2: Physical Data ${ }^{1}$.

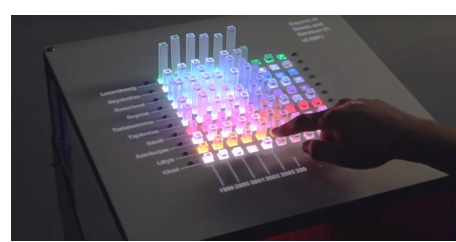

Figure 3: EMERGE [15]. a Physicalization as "a physical artifact whose geometry or material properties encode data" and propose to think of Data Physicalization as a research area that "examines how computer-supported, physical representations of data (i.e., physicalizations), can support cognition, communication, learning, problem solving, and decision making". Static Physicalizations are hard to modify, but the geometry or material properties of Dynamic Physicalizations can be modified (e.g. by computer-controlled actuators). Examples of dynamic physicalizations are physical charts, like the bar charts displayed with inFORM [3] (Figure 1), like the Physical Data ${ }^{2}$ (Figure 2), and like EMERGE [15] (Figure 3). As noted by Jansen et al. [7], "The growing popularity of data physicalizations in many societal domains indicates a strong potential for fostering public engagement. This popularity suggests that data physicalizations pique interest, and this interest could in turn be leveraged to have people spend more time and effort exploring and understanding important and complex data".

In this paper, we explain our interest in using dynamic physical charts to foster the shift of energy demand in public spaces. We design CairnFORM, a dynamic physical ring chart, for visualizing renewable energy forecasts. One advantage of these ring charts is to be readable at $360^{\circ}$. They can be used to display data in public spaces where people come and go to different directions such as entrance halls, roundabouts, public places, train stations and airports. We describe the design process of a first prototype of an expandable illuminated ring which is the basis component of the dynamic physical ring chart. Finally, we list some limitations of the current prototype and we evoke future works concerning the design and the study of CairnFORM.

\section{Using Dynamic Physical Charts to Foster the Shift of Energy Demand in Public Spaces}

Using renewable energy rather than nonrenewable energy is a strategy considered as appropriate for reducing environmental impact. However, as energy generation shifts to renewables and microgeneration ${ }^{1}$, the interplay between energy supply and energy demand becomes more complex to manage. Critical problems, such as peak demand, can lead to power outages and make the electrical grid inefficient [4]. These issues are amplified by renewable energy generation fluctuating with weather conditions (e.g. sun, wind, wave, tide) and by a limited energy storage capacity [5]. Unless energy storage becomes economically and environmentally reliable to balance energy supply against energy demand [10], we will need to synchronize the energy demand with the availability of renewables.

During the last five years, researchers have designed and studied systems to encourage users in using renewable energy when there is plenty of it rather than when there is little (i.e. shifting energy demand) [1, 8, 11, 12, 13, 14]. Most of the designed systems target households and help inhabitants to plan their everyday energy usage by visualizing renewable energy forecasts using graphical charts such as clock charts $[8,13]$, timeline charts [13] and line charts $[8,14]$.

To the extent of our knowledge, no dynamic physical chart has been used for visualizing renewable energy forecasts in a public space. Yet, as evoked by Zuckerman [17], tangible interfaces have strengths that can contribute to "change what people think and do" [2]: Visibility and Persistance; Situatedness; Tangible representation; and Affordances.

\footnotetext{
${ }^{1}$ Microgeneration is the small-scale generation of electricity and/or heat by individuals, businesses, or communities to meet their own needs. ${ }^{2}$ http://dataphys.org/list/dynamic-physical-charts-to-communicatecommunity-data/, accessed 13/09/2017.
} 


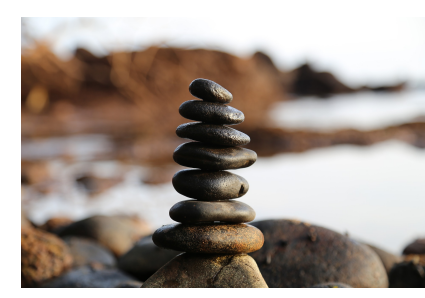

Figure 4: A cairn is a stack of stones indicating a landmark, a path, a border, or a sacred place ${ }^{3}$.

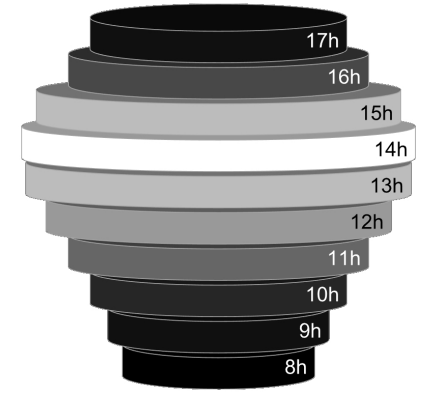

Figure 5: An illustration of CairnFORM, a dynamic physical ring chart.

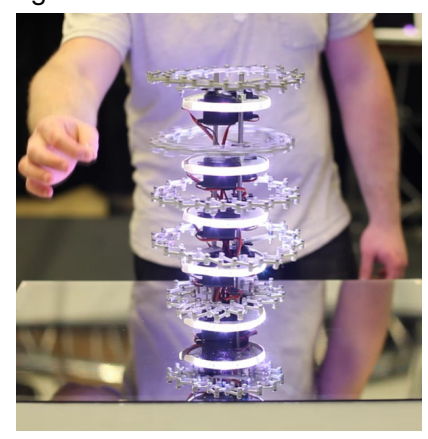

Figure 6: The actuated skeleton of AMPHORM (i.e. without its skin), a cylindrical kinetic sculpture [9].
Through these properties, tangible interfaces such as dynamic physical charts can help reducing the intangibility and the invisibility of energy that drives citizens away from energy [11].

\section{CairnFORM: a Dynamic Physical Ring Chart}

Bar charts are data visualizations commonly used to perform data comparisons over time. Therefore, bar charts seem relevant for highlighting trends in renewable energy forecasts such as incoming peaks of renewable energy. In the literature, we found examples of dynamic physical bar charts such as bar charts displayed with inFORM [3] (Figure 1), Physical Charts ${ }^{2}$ (Figure 2) and EMERGE [15] (Figure 3). However vertical bars are not readable at $360^{\circ}$ around because of occlusion effects. This occlusion effect can be countered by moving around the interface. Nonetheless, users can hardly move around the interface in many cases: train stations, public places, airports, roundabouts are public spaces where people are in a hurry and do not have time to change their point of view.

To counter this occlusion effect, we propose to use a vertical physical chart readable at $360^{\circ}$ around it. Inspired by stone cairns (Figure 4), we propose to design CairnFORM, a stack of expandable illuminated rings that can morph to different cairn shapes (i.e. defined by the number of rings and their diameter). For example, a ten-rings-CairnFORM can represent renewable energy forecasts for each hour of a workday (i.e. 8 am to $5 \mathrm{pm}$ ) (Figure 5). The ring's diameter and brightness would both be correlated to the average power forecasted to be generated in the hour: for a renewable power generation capacity ranging from $0 \mathrm{~W}$ to $\max \mathrm{W}$, a forecast of $0 \mathrm{~W}$ would be represented by a fully retracted ring with no brightness and a forecast of max $\mathrm{W}$ would be represented by a fully expanded ring with full brightness.

\section{Designing an Expandable Illuminated Ring}

The expandable illuminated ring is the basis component of CairnFORM. To design an expandable illuminated ring, we first looked for existing kinetic mechanisms allowing the expansion of a ring. In the literature, AMPHORM [9] makes use of expandable rings to shape a silicon skin: the result is a shape-changing vase (amphora). The aluminium rings of the skeleton of AMPHORM (Figure 6) are based on the Hoberman mechanism [6]. Based on three existing kinetic mechanisms, we iteratively prototyped a new expandable ring mechanism. Finally, we added illumination to the new expandable ring mechanism to obtain the current expandable illuminated ring. All along the design process, we eliminated expandable ring mechanisms that do not meet the following criteria: 1) the kinetic mechanism has inner-space available to put electronics (e.g. actuator, sensor) for actuation; 2) the kinetic mechanism maintains a regular ring-like shape during and after the expansion; and 3) the mechanism expands to a ring-like shape nearly two times larger than its initial size. We detail these steps in the following.

Existing Expandable Ring Mechanisms

We found three existing kinetic mechanisms allowing the expansion of a ring (Figure 7):

1. HobermanRing-7points ${ }^{4}$ (Figure 7a) and HobermanRing-12points ${ }^{5}$ (Figure $7 \mathrm{~b}$ ) are based on the Hoberman mechanism and can expand to a continuous ring-like shape two times larger than its initial size. However during the expansion, the ring shape changes in a seven-pointed star with HobermanRing-7points and in a twelve-pointed star

${ }^{3}$ https://pixabay.com/p-2082937, image under CC0 licence, downloaded 14/09/2017. 


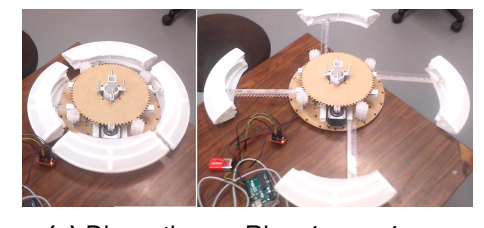

(a) DiscontinuousRing-4arms-4arcs

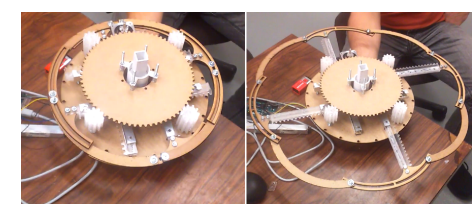

(b) IrregularRing-4arms-8arcs

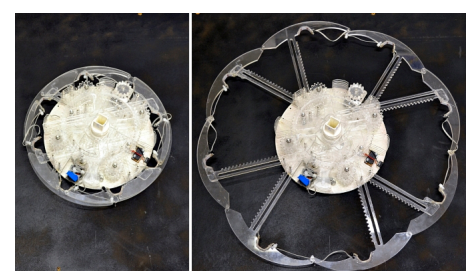

(c) RingLike-8arms-8arcs

Figure 8: The three expandable ring mechanisms made during the iterative prototyping.

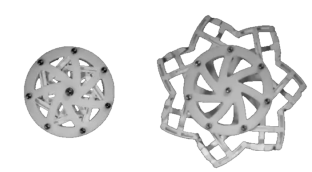

(a) HobermanRing-7points ${ }^{4}$

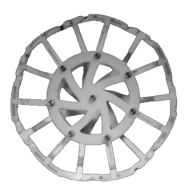

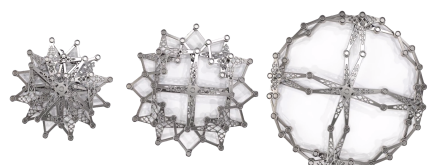

(b) HobermanRing-12points ${ }^{5}$

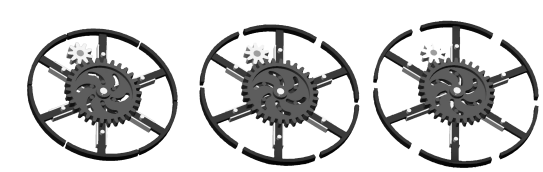

(c) DiscontinuousRing-6arms-6arcs ${ }^{6}$

Figure 7: Three existing expandable ring mechanisms.

with HobermanRing-12points. Moreover, very few inner-space is available.

2. The structure DiscontinuousRing-6arms-6arcs ${ }^{6}$ (Figure 7c) maintains its ring-like shape during the expansion and there is inner-space available. However, it only expands to a discontinuous ring-like shape less than two times larger than its initial size.

Iterative prototyping of a new expandable ring mechanism Through iterative prototyping, we designed a new expandable ring mechanism. Based on DiscontinuousRing6-6 and inspired by HobermanRing-7/12, we prototyped three different expandable ring mechanisms before obtaining the current version:

1. DiscontinuousRing-4arms-4arcs (Figure 8a) can expand to a shape three times larger than its initial size. However, during the expansion, the ring shape changes from a continuous ring to a discontinuous rounded square.

${ }^{4}$ https://vimeo.com/32823353, accessed 13/09/2017.

$5 \mathrm{https}: / /$ www.youtube.com/watch?v=5eOG-uEqDxU, accessed 13/09/2017.

${ }^{6} \mathrm{https}: / /$ www.youtube.com/watch?v=KgYX9m6sG5c, accessed 13/09/2017.
2. IrregularRing-4arms-8arcs (Figure 8b) can expand to a continuous ring-like shape two times larger than its initial size. However during the expansion, the ring shape changes from a continuous regular ring to a continuous irregular ring-like shape.

3. RingLike-8arms-8arcs (Figure 8c) can expand to a continuous ring-like shape two times larger than its initial size while maintaining a regular ring-like shape.

Adding illumination to the new expandable ring mechanism Based on the RingLike-8arms-8arcs mechanism (Figure 9), our final prototype of an expandable illuminated ring (Figure 10) is composed of a column making the ring stackable. The ring is actuated by a TRINAMIC stepper motor QSH4218-41-10-035 with a transmission gear that activates a wheel gear. The wheel activates four pinion gears. Each pinion gear activates an axial rack and a diagonal rack. At the edge of each rack, there is an arc representing one eighth of a $\varnothing 35 \mathrm{~cm}$ ring. When the ring is fully retracted, the arcs form the initial ring $(\varnothing 35 \mathrm{~cm})$. When the ring is fully expanded, the arcs form a ring-like shape nearly two times larger than its initial size $(\varnothing 62 \mathrm{~cm})$. The ring is illuminated by 24 NeoPixel Digital RGB LEDs (three LEDs on each arc). Furthermore, a micro-switch is used to sense when the ring is fully retracted. 


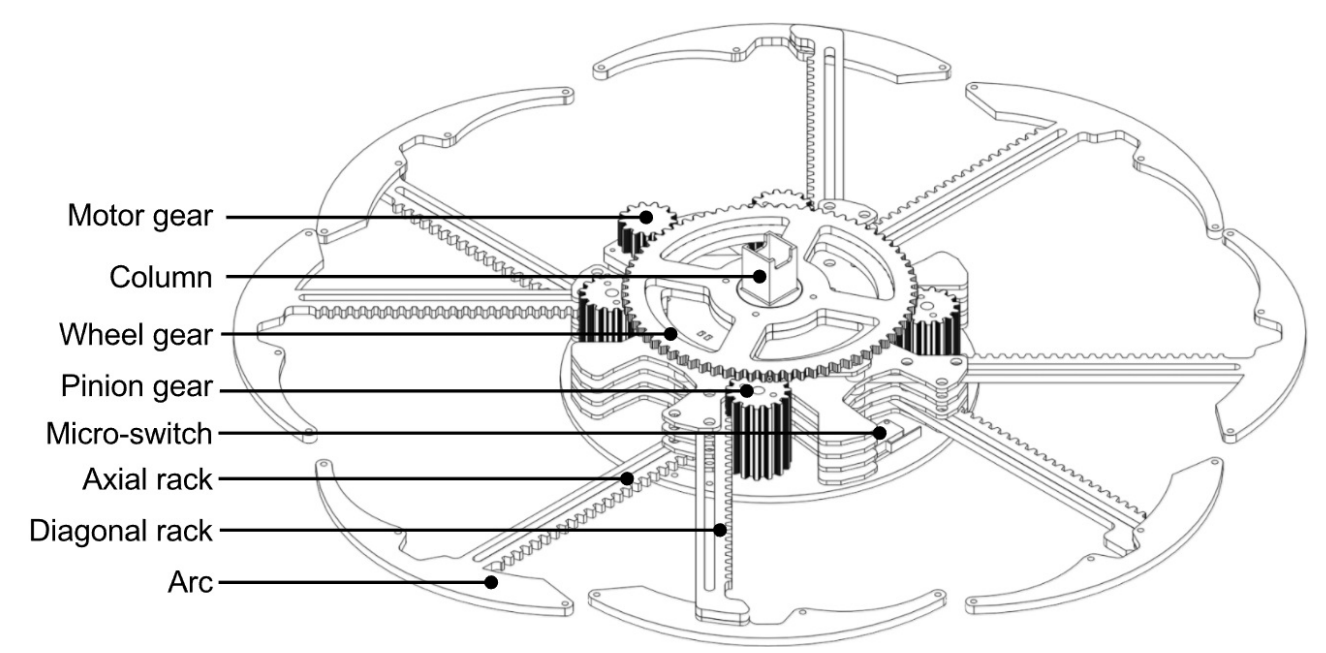

Figure 9: The CAD model of the mechanism of the final expandable ring $^{6}$, designed with OnShape, a Cloud CAD Software.

The column was manufactured in PLA material using 3D printing. All other parts were made in translucent PMMA material using 2D laser cutting and assembled using

acetone. The remaining parts, mainly support parts, were made in plywood using 2D laser cutting. Bolts, nuts and washers were used to assemble all the parts together.

All LEDs and the stepper motor are powered by a ATX computer power supply. The 24 LEDs are controlled using an Arduino Mega2560. The stepper motor is controlled using an Adafruit stepper motor HAT for Raspberry Pi. The micro-switch is read using a pull-up circuit on an Adafruit perma-proto HAT for Raspberry Pi. One Raspberry Pi 3 is used to communicate with the Mega2560 (serial protocol) and the HATs (I2C protocol). The driver is written in Python 2.7.

\section{Limitations}

We identified four limitations of the current prototype:

- Expansion range. The current prototype expands with a 1.8 diameter scale factor (ranging from $35 \mathrm{~cm}$ to $62 \mathrm{~cm}$ ). The smallest the expansion range is, the more the difference between each ring of the final ring chart will be subtle to perceive. In our case, the expansion range is $27 \mathrm{~cm}$ : to display a 256-point data scale, the granularity would be only $\sim 1 \mathrm{~mm}$, would be $\sim 3 \mathrm{~mm}$ for a 100-point data scale, and would more reasonably be $\sim 1 \mathrm{~cm}$ for a 30-point data scale.

${ }^{6}$ The CAD model is available at: https://cad.onshape.com/documents/ 7d4ecae370a1e03250f148cc/w/40919688efd268263900abc1/e/ 6c37dd97791037a37d7dd39b, accessed 13/09/2017. 

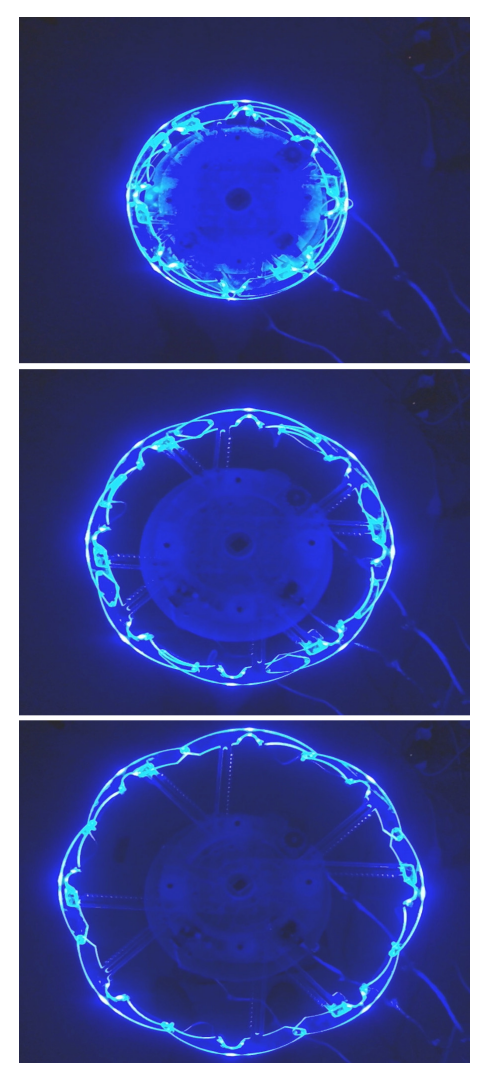

Figure 10: The final prototype of an expandable illuminated ring.
- Motion noise. When the ring is expanding, the overall structure generates about $60 \mathrm{~dB}$ at a distance of 1 meter, when the $5 \mathrm{~V}$ stepper motor QSH4218 is at 60 RPM in double coil mode. This noise makes the current ring intrusive: it should be used in situations where noise does not reduce usability (e.g. in an entrance hall, but not in an office or an open plan).

- Power consumption. In the worst case, the prototype draws up to $12.2 \mathrm{~W}$ (7.2 W max for LEDs and $5 \mathrm{~W}$ max for the stepper motor). This case occurs when actuating the ring at full brightness with the stepper motor in double coils mode (the most energy-consuming mode). This case will rarely or never occur. However, because the purpose of the final ring chart will be to engage users with an energyrelated issue, this chart will have to help in saving more energy than it consumes.

- Fabrication cost. A ring costs about $200 €$ to build: $60 €$ of raw materials (PLA, PMMA, and plywood) manufactured using a 3D printer and a $2 \mathrm{D}$ laser cutter; $100 €$ of electronics (NeoPixel digital RGB LED, stepper motor QSH4218, and micro-switch); and $40 €$ of bolts, nuts, washers, aluminium profiles, and other products such as PVC glue and acetone. A prototype stacking ten expandable illuminated rings will cost approximately $2000 €$ for the rings plus $300 €$ of electronics to control it (Raspberry Pi 3, Adafruit perma-proto HATs, Adafruit stepper motor HATs, Arduino Mega2560, and ATX power supply).

\section{Conclusion and Future Works}

In this paper, we propose to use dynamic physical charts to foster shifting energy demand in public spaces. We think that the tangible properties [17] of physical charts such as the Visibility and Persistance, the Situatedness and the Tangible representation can help mitigating the intangibility and the invisibility of energy that drives citizens away from energy [11]. Therefore, we propose to use a dynamic physical ring chart for visualizing renewable energy forecasts. We propose the design of CairnFORM, a customizable stack of expandable illuminated rings readable at $360^{\circ}$ around it. Inspired by existing kinetic mechanisms, we describe the design process of a first prototype of an expandable illuminated ring. Furthermore, we identify several limitations of our final prototype of an expandable illuminated ring such as the expansion range, the motion noise, the power consumption, and the fabrication cost.

In future works, we will manufacture 10 expandable illuminated rings. With this stack of 10 rings, we will conduct in vitro user studies to assess the readability of ring charts when asking users to retrieve incoming peaks of renewable energy production. Furthermore, we will study in vivo the attractiveness over weeks and months of such dynamic physical charts in a public space.

\section{References}

[1] Enrico Costanza, Joel E. Fischer, James A. Colley, Tom Rodden, Sarvapali D. Ramchurn, and Nicholas R. Jennings. 2014. Doing the Laundry with Agents: A Field Trial of a Future Smart Energy System in the Home. In Proceedings of the SIGCHI Conference on Human Factors in Computing Systems (CHI '14). ACM, New York, NY, USA, 813-822. DOI : http://dx.doi.org/ 10.1145/2556288.2557167

[2] Brian J. Fogg. 1998. Persuasive Computers: Perspectives and Research Directions. In Proceedings of the SIGCHI Conference on Human Factors in Computing Systems (CHI '98). ACM Press/Addison- 
Wesley Publishing Co., New York, NY, USA, 225-232. DOI : http://dx.doi.org/10.1145/274644.274677

[3] Sean Follmer, Daniel Leithinger, Alex Olwal, Akimitsu Hogge, and Hiroshi Ishii. 2013. inFORM: Dynamic Physical Affordances and Constraints Through Shape and Object Actuation. In Proceedings of the 26th Annual ACM Symposium on User Interface Software and Technology (UIST '13). ACM, New York, NY, USA, 417-426. DOI : http://dx.doi.org/10.1145/2501988. 2502032

[4] Frontier Economics and Sustainability First. 2012. Demand side response in the domestic sector-a literature review of major trials. UK Department of Energy and Climate Change, Final Report, London, August. (2012). http: //www.frontier-economics.com/documents/2013/10/ frontier-report-demand-side-response-in-the-domestic-sector. pdf Accessed 13/06/2017.

[5] David Hafemeister. 2010. Sustainable Energy - Without the Hot Air. American Journal of Physics 78, 2 (2010), 222-223. DOI : http://dx.doi.org/10.1119/1.3273852

[6] Charles Hoberman. 1991. Radial expansion/retraction truss structures. (June 1991). US Patent 5,024,031.

[7] Yvonne Jansen, Pierre Dragicevic, Petra Isenberg, Jason Alexander, Abhijit Karnik, Johan Kildal, Sriram Subramanian, and Kasper Hornbæk. 2015. Opportunities and Challenges for Data Physicalization. In Proceedings of the 33rd Annual ACM Conference on Human Factors in Computing Systems $(\mathrm{CHI}$ '15). ACM, New York, NY, USA, 3227-3236. DOI: http://dx.doi.org/10.1145/2702123.2702180

[8] Jesper Kjeldskov, Mikael B. Skov, Jeni Paay, Dennis Lund, Tue Madsen, and Michael Nielsen. 2015. Facilitating Flexible Electricity Use in the Home with
Eco-Feedback and Eco-Forecasting. In Proceedings of the Annual Meeting of the Australian Special Interest Group for Computer Human Interaction (OzCHI '15). ACM, New York, NY, USA, 388-396. DOI : http://dx.doi.org/10.1145/2838739.2838755

[9] David Lakatods. 2012. AMPHORM: Form Giving through Gestural Interaction to Shape Changing Objects. Master's thesis. Massachusetts Institute of Technology (MIT), Cambridge, MA, USA.

[10] Xing Luo, Jihong Wang, Mark Dooner, and Jonathan Clarke. 2015. Overview of current development in electrical energy storage technologies and the application potential in power system operation. Applied Energy 137 (January 2015), 511-536. http://wrap.warwick.ac.uk/63615/

[11] James Pierce and Eric Paulos. 2010. Materializing Energy. In Proceedings of the 8th ACM Conference on Designing Interactive Systems (DIS '10). ACM, New York, NY, USA, 113-122. DOI : http://dx.doi.org/10. 1145/1858171.1858193

[12] Filipe Quintal, Clinton Jorge, Valentina Nisi, and Nuno Nunes. 2016. Watt-I-See: A Tangible Visualization of Energy. In Proceedings of the International Working Conference on Advanced Visual Interfaces (AVI '16). ACM, New York, NY, USA, 120-127. DOI : http://dx.doi. org/10.1145/2909132.2909270

[13] Johann Schrammel, Cornelia Gerdenitsch, Astrid Weiss, Patricia M. Kluckner, and Manfred Tscheligi. 2011. FORE-Watch - The Clock That Tells You When to Use: Persuading Users to Align Their Energy Consumption with Green Power Availability. In Proceedings of the 2nd International Conference on Ambient Intelligence (Aml '11). Springer Berlin Heidelberg, Berlin, Heidelberg, 157-166. DOI : http://dx.doi.org/10.1007/978-3-642-25167-2_19 
[14] Will Simm, Maria Angela Ferrario, Adrian Friday, Peter Newman, Stephen Forshaw, Mike Hazas, and Alan Dix. 2015. Tiree Energy Pulse: Exploring Renewable Energy Forecasts on the Edge of the Grid. In Proceedings of the SIGCHI Conference on Human Factors in Computing Systems (CHI '15). ACM, New York, NY, USA, 1965-1974. DOI : http://dx.doi.org/10.1145/2702123.2702285

[15] Faisal Taher, John Hardy, Abhijit Karnik, Christian Weichel, Yvonne Jansen, Kasper Hornbæk, and Jason Alexander. 2015. Exploring Interactions with Physically Dynamic Bar Charts. In Proceedings of the 33rd Annual ACM Conference on Human Factors in Computing Systems (CHI '15). ACM, New York, NY, USA, 3237-3246. DOI : http://dx.doi.org/10.1145/ 2702123.2702604

[16] Brygg Ullmer and Hiroshi Ishii. 2000. Emerging Frameworks for Tangible User Interfaces. IBM Syst. J. 39, 3-4 (July 2000), 915-931. DOI : http: //dx.doi.org/10.1147/sj.393.0915

[17] Oren Zuckerman. 2015. Objects for Change: A Case Study of a Tangible User Interface for Behavior Change. In Proceedings of the Ninth International Conference on Tangible, Embedded, and Embodied Interaction (TEI '15). ACM, New York, NY, USA, 649654. DOI : http://dx.doi.org/10.1145/2677199.2687906 